\title{
Enhanced Quality of Service Based Routing Protocol Using Hybrid Ant Colony Optimization and Particle Swarm Optimization
}

\author{
Neelam Kumari \\ Dept. Computer Science and Engineering \\ Beant College of Engineering \& Technology
}

\author{
Arpinder Singh Sandhu \\ Dept. Computer Science and Engineering \\ Beant College of Engineering \& Technology
}

\begin{abstract}
The main problem of QoS routing is to setup a multicast hierarchy that may meet particular QoS constraint. In order to reduce the constraints of the earlier work a new improved technique is proposed in this work. In the proposed technique the issue of multi-cast tree is eliminated using clustering based technique. First of all multi-radio and multichannel based clustering is deployed and these cluster head are responsible for the multicasting. It will diminish the overall energy consumption of nodes and complexity of intelligent algorithms. The path will be evaluated based upon the ant colony optimization. Thus it has produced better results than other techniques.
\end{abstract}

Keywords: QoS, Multicast, Ant colony optimisation, clustering.

\section{Introduction}

A Mobile Ad-hoc Network is an accumulation of independent mobile nodes that can communicate together via Radio Lake. Your mobile nodes which has been in radio selection of each various other could right communicate, whereas others needs the aid of intermediate nodes to route his or her packets. The entire node carries a radio user interface to connect jointly. These networks usually are fully distributed, and perform at any place without the aid of any fixed infrastructure as gain access to points or base areas. Figure 1 shows a simple ad-hoc network having 3 nodes. Node 1 in addition to node 3 isn't within range of each and every other, however the node 2 enables you to forward packets between node 1 and node a couple of. The node 2 will behave as a router in addition to these three nodes in concert form an ad-hoc system.

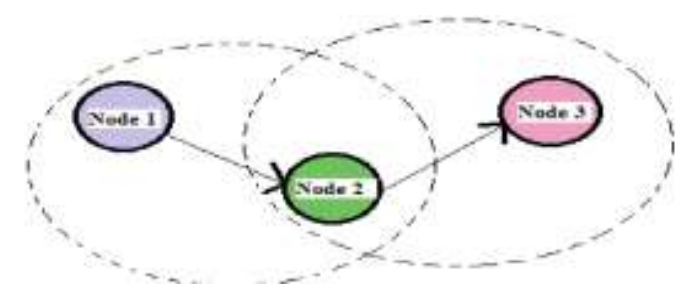

Fig. 1 Example of mobile ad-hoc network

\subsection{MANETs characteristics}

1) Dispersed operation: There isn't background network for that central control in the network operations; the control in the network is distributed one of the nodes. The nodes associated with a MANET should cooperate with each other and communicate among themselves and each and every node acts to be a relay as essential, to implement specific functions for example routing and security.

2) Multi get routing: When a node endeavours to send facts to other nodes that is out of its communication range, the packet the packet need to be forwarded via one or more intermediate node. 3) Autonomous fatal: In MANET, each mobile node is an independent node, which will function as both a host and a router.

4) Vibrant topology: Nodes are unengaged to move arbitrarily using different speeds; so, the network topology might change randomly and also at unpredictable time. The nodes in the MANET dynamically determine routing among themselves since they travel around, establishing their unique network.

5) Light-weight terminals: Within maximum cases, the actual nodes at MANET are generally mobile with much less CPU capability, minimal power storage and also small memory measurement.

6) Shared Physical Medium: The wireless communication medium is obtainable to any entity with all the appropriate equipment and also adequate resources. Keeping that in mind, access to the channel is not restricted.

\section{Advantages of MANET}

Why people love an Ad-Hoc network add the following:

- They feature access to information and services no matter geographic position.

- Liberty from central circle administration. Self-configuring circle, nodes are also behave as routers. Less expensive compared to wired network.

- Scalable - accommodates the particular addition of additional nodes. 
International Journal of Computer Applications Technology and Research

Volume 4- Issue 8, 618 - 622, 2015, ISSN: 2319-8656

- Much better Flexiblibility.

- Robust on account of decentralize administration.

- The network might be set up at any place and time.

\subsubsection{MANETs Challenges}

1)Limited bandwidth: Wireless link keep have significantly reduced capacity than infrastructure networks. In addition, the realized throughput involving wireless communication after accounting for that effect of a number of accesses, fading, noise, and interference problems, etc., is often a reduced amount of than a radio's highest transmission rate.

2) Energetic topology: Dynamic topology member's program may disturb the particular trust relationship amongst nodes. The trusts are often disturbed if a number of nodes are recognized as compromised.

3) Course-plotting Overhead: In Wi-Fi ad-hoc networks, nodes frequently change their area within network. And so, some stale routes are generated from the routing table that leads to unnecessary direction-finding overhead.

4) Undetectable terminal problem: The hidden terminal problem describes the collision of packets with a receiving node a result of the simultaneous transmission of the nodes that aren't within the direct transmission selection of the sender, but are within the transmission range on the receiver.

5) Bundle losses on account of transmission mistakes: Ad hoc Wi-Fi networks experiences a far more achievable bundle loss due to factors like while increased collisions a consequence of the presence of cannot be seen terminals, presence involving disturbance, unidirectional links, frequent way breaks due to mobility associated with nodes.

6) Mobility-induced approach changes: The system topology inside the ad hoc Wi-Fi network can be highly dynamic a consequence of the activity of nodes; for that reasons an on-going interval suffers typical path pauses. This situation often leads to frequent way alterations.

7) Battery demands: Devices used throughout these networks have restrictions for the power source so as to maintain portability, size and weight on the device. 8) Security threats: The wireless mobile random nature of MANETs provides new security challenges for the network design. For the reason that wireless medium is at risk of eavesdropping and random network functionality is made through node assistance, mobile ad hoc networks are intrinsically confronted with numerous security attacks.

\subsubsection{MANETs Applications}

1) Military battlefield: Ad-Hoc networking will allow the military to reap the benefits of commonplace network technology to help keep an information network relating to the soldiers, vehicles, and military information brain quarter.

Unknown terrain

Limit the Range of communication

Directional Antennas
Destroyed infrastructure

2) Collaborative perform: For some enterprise environments, the need regarding collaborative computing may very well be more important outside office environments when compared with inside and in which people do must have outside meetings to help cooperate and exchange information on a given challenge.

3) Local levels: Ad-Hoc networks can autonomously link instantaneously and temporary media network using notebook computers to spread and share information among participants for a e. g. meeting or classroom. Another appropriate community level application may very well be in home networks where devices can communicate straight to exchange information.

4) Personalized area network and bluetooth: A personal area network is a short range, localized network in which nodes are usually associated with a given person. Short-range MANET like Bluetooth can de-stress the inter verbal exchanges between various mobile devices as being a laptop, and also a mobile phone.

5) Commercial Market: Ad hoc may be used in emergency/rescue procedures for disaster relief efforts, e. gary the gadget guy. in fire, avalanche, or earthquake. Emergency rescue operations must be held where non-existing or even damaged communications national infrastructure and rapid deployment of any communication network is necessary.

\subsection{SAFETY GOALS in MANET}

All networking functions for instance routing and bundle forwarding, are performed by nodes themselves in a very selforganizing manner. Therefore, securing a cell ad -hoc network is quite challenging. The goals to evaluate if mobile ad-hoc circle is secure or even not are as follows:

1)Availability: Availability suggests the assets are generally accessible to sanctioned parties at suitable times. Availability applies both to data in order to services. It makes sure the survivability associated with network service despite denial of services attack.

2) Privacy: Confidentiality ensures in which computer-related assets are generally accessed only by authorized parties. Protection of information which is exchanging through any MANET. It ought to be protected against any disclosure attack including eavesdropping- unauthorized examining of message.

3) Honesty: Integrity means that assets is usually modified only by authorized parties or even only in sanctioned way.. Integrity assures which a message being transported is never damaged.

4) Authentication: Authentication is defined as assurance men and women in communication are authenticated in lieu of impersonators. The recourses involving network needs to be accessed because of the authenticated nodes.

5) Acceptance: This property assigns distinct access rights to different types of users. For example any network management can be carried out by network officer only.

6) Strength to attacks: It must sustain the network functionalities whenever a portion of nodes is actually compromised or ruined. 
Volume 4- Issue 8, 618 - 622, 2015, ISSN: 2319-8656

7) Freshness: It ensures that malicious node doesn't resend previously seized packets.

\subsection{ROUTING PROTOCOLS}

Ad-Hoc circle routing protocols are commonly divided into about three main classes; Proactive, reactive and cross protocols as found in figure 2 .

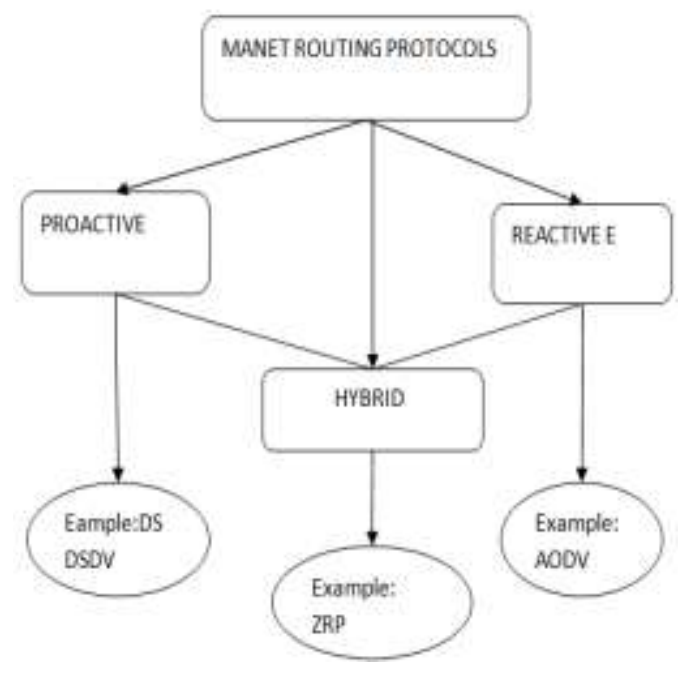

Fig. 2 Classification of MANET

\section{routing protocol}

Proactive Protocols: Proactive, or table-driven routing protocols. In positive routing, each node needs to maintain a number tables to shop routing information, and any alterations in network topology have to be reflected by propagating updates through the entire network to be able to maintain a regular network view. Example of such schemes include the conventional routing techniques: Destination sequenced mileage vector (DSDV). They attempt to maintain consistent, up-to-date routing information in the whole network. It minimizes your delay in communication and invite nodes to rapidly determine which nodes are present or reachable within the network.

Example: Destination-sequenced distance vector (DSDV), Wireless routing protocol (WRP), Global state routing (GSR), OLSR (Optimized Link State Routing)

Reactive Protocols: Reactive routing is also called on-demand routing protocol since they just don't maintain routing data or routing activity on the network nodes when there is no communication. If a node would like to send a packet completely to another node then this protocol pursuit of the route within the on-demand manner and establishes the connection to be able to transmit and receive the packet. The route finding occurs by inundating the route request packets through the entire network. Examples of reactive routing protocols include the Ad-hoc Ondemand Range Vector routing (AODV) and Dynamic Source routing (DSR).
Hybrid Protocols: They features a hybrid type that combines reactive and proactive routing standards. The Zone Courseplotting Protocol (ZRP) is often a hybrid routing process that divides your network into areas and specific zones. ZRP provides any hierarchical architecture where by each node needs to maintain additional topological data requiring extra memory space. Example: Zone routing protocol (ZRP),Distributed dynamic routing (DDR)

\section{Related Work}

Rajeev Agrawal (2001) [1] has adopted probabilistic modeling to model the effect due to multipath fading and shadowing. The BER for each link affected by the fading is estimated using the proposed model. Wireless Routing Protocol (WRP) maintains the BER associated with a particular link, a packet/ data is routed with optimum BER route from a set of discovered route by protocol. B.Malarkodi et al. (2009) [2] the impact of different mobility models on Multicast Routing Protocols. The results showed that the throughput of ADMR is higher than of ODMRP at high mobility. This is achieved at the cost of increase in delay and transmission over head. Under low mobility, ODMRP has higher throughput than AMDR.

V.A Gajbhiye and R. W.Jasutkar(2013) [3] showed that Swarm Intelligence based routing protocol has shown promising results in VANET. For this they compared and evaluated the performance of AODV, OLSR, and Swarm Intelligence based routing protocol in terms of throughput, latency and data packet delivery ratio for VANET. Simulation results have shown that SWARM Intelligence based routing protocol showed promising results in VANETs as compared to AODV and OLSR.

Nathaniel Gemelli et al. (2003) [4] Introduced Bluetooth wireless technology, examine current routing protocols and present the objectives and considerations for the design of a new Bluetooth routing protocol. The protocol design would consider the capabilities of the devices (nodes) within the range of the network. It was envisioned that capabilities Aware Routing (CAR) protocol would make routing decisions based on such. Factors as device power constraints E.Ahila Devi and K.Chitra(2014 )[5] Introduced a Privacy Protecting Secure and Energy Efficient Routing Protocol (PPSEER) was proposed. In this protocol, first the classifications of network node take place based on their energy level.

Hiba Hachichi et al. (2011) [6] created and maintained locally a hierarchy that was well suitable for routing packets in an Ad hoc network. The contribution of this work was mainly based on the construction of a virtual topology where cluster heads and gateways collaborate for searching the destination node.

Istikmal et al. (2013) [7] presented about investigation result of AODV, DSR and DSDV that applied an Ant-algorithm which were AODV-Ant, DSR-Ant, and DSDV-Ant. DSDV represents of proactive routing type protocol based on table driven, while AODV and DSR represents of reactive routing protocol type based on demand. Performance analysis included end to end delay, throughput, routing overhead and hop count for various scenario of node velocity, pause time and network traffic. 
Volume 4- Issue 8, 618 - 622, 2015, ISSN: 2319-8656

Sikkandar Ali and Vashik Ali et al.. (2012)[8] presented routing in wireless mobile ad-hoc networks using Destination Sequenced Distance Vector (DSDV) and Ad-hoc on demand Distance Vector (AODV) protocols. The performance of bandwidth, throughput and packet loss of DSDV and AODV has been modelled under various network configurations and mobility conditions.

Geethu Mohandas(2013) [9] The Mobile Ad hoc Networks (MANET) are networks with self-configuring capacity of mobile devices interconnected by wireless links. During the last few years, research in various aspects of MANETs has been prominent, prompted mainly by military, disaster relief, and law enforcement scenarios. An instinctive footstep was to take up such location-based operation to MANETS.

KomalPatel et al.(2006)[10] proposed a cross layer approach that uses the MAC layer link stability information to improve the routing efficiency. Signal strength of the link was captured from the MAC Layer and used at network layer to predict the future signal strength value using double exponential smoothing model. This information was used to categorize the link as stable or unstable.

Rashmi Rohankar et al. (2012) [11] analyzed the effect of random based mobility models on the performance of Proactive Routing Protocol (DSDV Destination Sequence Distance Vector) and Reactive Routing Protocol (AODV- on Demand Distance Vector, DSR- Dynamic Source Routing). Performance analysis was done with respect to end-to-end delay, throughput and Packet delivery ratio for varying node densities.

Yudhvir Singh(2010)[12] performed simulation based experiments were performed to analyzed the performance of On Demand Multicast Routing Protocol by evaluating Packet Delivery Ratio, End to End delay and average throughput. These results were compared with AODV and FSR routing protocols by varying number of nodes and mobility. The comparison showed that ODMRP for ad hoc networks performs better as compared to AODV and FSR.

\section{Proposed Technique}

Following are the various steps required to successfully simulate the proposed algorithm.

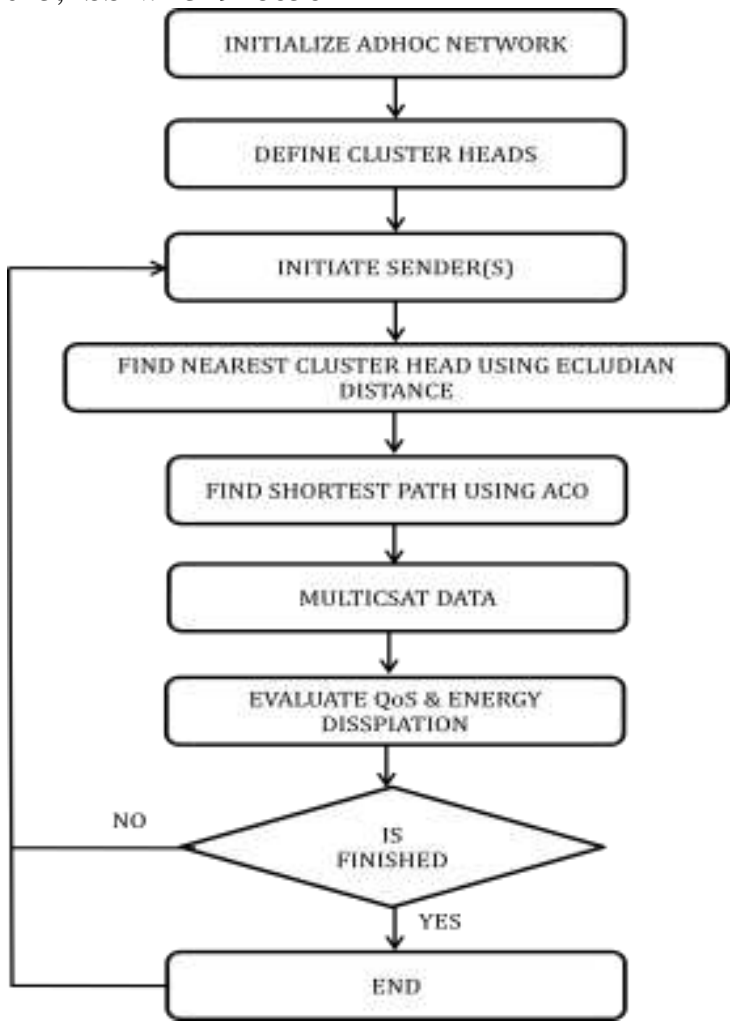

Figure 1: Flowchart of the proposed technique

Step 1: First of all initialize ad-hoc network with their respective characteristics like moving range, maximum dimensions, number of nodes etc.

Step 2: Define cluster heads having multi-radio and multichannel facility.

Step 3: Sender(s) will be initiated to multicast its data to defined nodes.

Step 4: Sender will hand over its data to nearest cluster head using Euclidian distance.

Step 5: Cluster head will multicast data to available cluster heads depends upon the ACO based shortest path.

Step 6: Evaluate energy dissipation as well as other QoS features, and move to step 3

\section{Result Analysis}

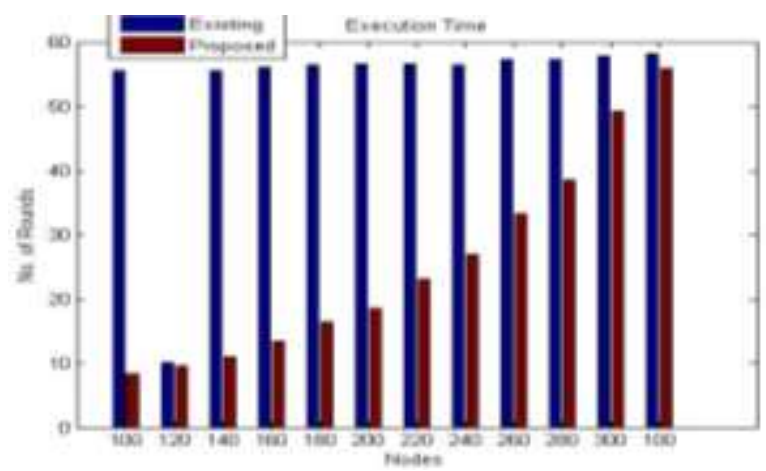

fiFig1: Execution Time 
The Figure represent the information about the Execution time of Existing and Proposed Technique $\mathrm{X}$-axis signify the value of number of Nodes and Y-axis correspond to the value of Number of Rounds. Moreover, in which two type of color are used i.e Red and Blue. Red color symbolize the Proposed technique and Blue color symbolize the Existing Technique.

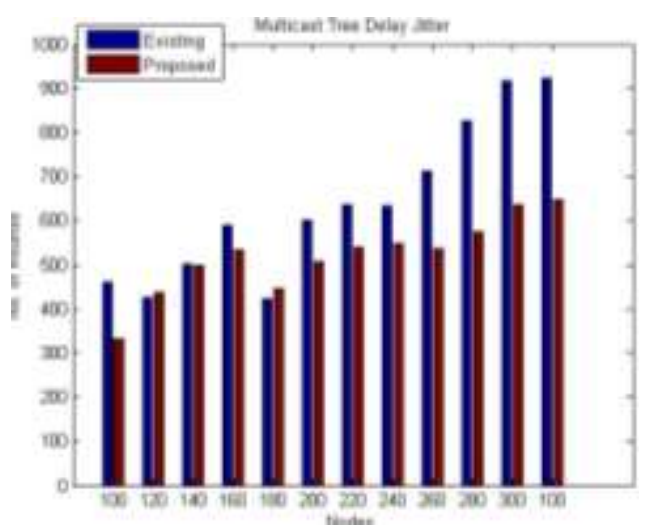

Fig2: Multicast Tree Delay Jitter

The Diagram represent the information about the Multicast Tree Delay Jitter of Existing and Proposed Technique $\mathrm{X}$-axis signify the value of number of Nodes and Y-axis correspond to the value of Number of Rounds. Moreover, in which two type of color are used i.e Red and Blue. Red color symbolize the Proposed technique and Blue color symbolize the Existing Technique.

\section{Conclusion}

The main problem of QoS routing is to setup a multicast hierarchy that may meet particular QoS constraint. Nevertheless, the situation of making a multicast tree below several constraints is available to be NP Complete. Therefore, the issue is often settled by heuristics or smart optimization. Lately, some meta-heuristic algorithms including the ant colony algorithm, genetic algorithm and compound swarm optimization have been employed by the analysts to eliminate the multi-constrained QoS routing problem. In order to reduce the constraints of the earlier work a new improved technique is proposed in this work. In the proposed technique the issue of multi-cast tree is eliminated using clustering based technique. First of all multi-radio and multichannel based clustering is deployed and these cluster head are responsible for the multicasting. It will diminish the overall energy consumption of nodes and complexity of intelligent algorithms. The path will be evaluated based upon the ant colony optimization. Thus it has produced better results than other techniques.

This work has not considered the effect of node failures on the network. Therefore in near future we will evaluate the node failures while data communication is in progress.

\section{REFERENCES}

[1] Agrawal, Rajeev. "Performance of routing strategy (bit error based) in fading environments for mobile adhoc networks." IEEE International Conference on Personal Wireless Communications, pp. 550-554, 2005.

[2] Malarkodi, B.P. Gopal and B.Venkataramani. "Performance Evaluation of Adhoc Networks with Different Multicast Routing Protocols and Mobility Models." IEEE International Conference on advanced in recent technologies in communication and computing, pp. 81-84, 2009.

[3] Gajbhiye, V. A and R. W. Jasutkar. "Biologicaly inspired routing protocol for vehicular ad hoc network." $6^{\text {th }}$ IEEE International Conference on Advanced Infocomm Technology, pp. 202-206, 2013.

[4] Gemelli, Nathaniel, Peter LaMonica,Paul Prtzke, John Spina."Capabilities aware routing for dynamic ad hoc networks." IEEE International Conference on Integration of knowledge Intensive Multi-Agent Systems, pp. 585-590, IEEE, 2014. 2003.

[5] Devi, E.Ahila, and K.Chitra. "Security based energy efficient routing protocol for Ad hoc network." IEEE International Conference on Control, Instrumentation, Communication and Computational Technologies, pp. 1522-1526, 2014.

[6] Hachichi, Hiba, Samia Chelloug and Fatima Athmouni. "A virtual topology for routing in adhoc networks." IEEE International Conference on Electronics, Communication \& Phtonics, 2011.

[7] Ali, Sikkandar AliVashik, W.R.Salem Jeyaseelan and Shanmugasundaram Hariharan. "Enhanced Route Discovery in Mobile Adhoc Networks." IEEE Third International Conference on. Computing Communication and Networking Technologies, pp. 1-5,2012.

[8] Istikmal,Leanna, V. Y and Basuki Rahmat. "Comparison of proactive and reactive routing protocol in mobile ad hoc network based on "Ant-algorithm." IEEE International Conference on Computer, Control, Informatics and its Applications, 2013.

[9] Mohandas, Geethu, Salaja Silas and Shini Sam. "Survey on routing protocols on mobile ad hoc networks." IEEE International Multi-Conference on Automation, Computing, Communication, Control and Compressed Sensing, pp. 514517,2013

[10] Patel, Komal, S. Srivastava and R. B. Lenin. "MAC Layer aware Stable Link Routing (MACSLR) in Mobile Ad hoc Networks." IEEE International Symposium on Ad hoc and Ubiquitous Computing, pp. 298-301, 2006.

[11] Rohankar, Rashmi, Rinkoo Bhatia and Deepak Kumar Sharma."Performance analysis of various routing protocols (proactive and reactive) for random mobility models of Ad hoc networks." It IEEE International Conference on Recent Advance in Information Technology, 2012.

[12] Yudhvir Singh, Yogesh Chaba, Monica Jain and Praba Rani. "Performance Evaluation of On-Demand Multicasting Routing Protocols in Mobile Adhoc Networks." IEEE, International Conference on Recent Trend in Information, Telecommunication \& Computing, pp.298-301, 2010. 